\title{
Re: Machine Perfusion of Donor Kidneys May Reduce Graft Rejection
}

\author{
Samoylova ML1, Nash A1, Kuchibhatla M22, Barbas AS1, Brennan TV3 \\ 'Duke University Faculty of Medicine, Department of Surgery, North Carolina, US \\ 2Duke University Faculty of Medicine, Department of Biostatistics, North Carolina, US \\ ${ }^{3}$ Cedars-Sinai Faculty of Medicine, Department of Surgery, California, US
}

Clin Transplant. 2019 Sep 21:e13716. doi: 10.1111/ctr.13716. [Epub ahead of print]

\section{EDITORIAL COMMENT}

Hypothermic machine perfusion (MP) is increasingly being used to expand the marginal donor pool to decrease the rates of delayed graft function (DGF), especially for those kidneys procured from high kidney donor risk index (KDRI) donors and deceased after cardiac death (DCD) donors. Continuous MP of the donor kidney can lead to reduced tubular and endothelial injury and better glomerular function when compared to static cold storage. Monitoring of intravascular graft resistance by measuring the perfusion pressures and the perfusate flow during MP also allows delivery of vasoactive agents that may potentially improve early graft function. However, there is very limited data on MP and allograft rejection. In this retrospective cohort study, the authors have evaluated the effects of MP on allograft rejection in 79.300 kidney-alone transplant recipients of whom 42\% have undergone MP over a 13-year period in the United States. MP kidneys came from older donors, were more likely to have been obtained following DCD, and had longer cold ischemic times. Although rates of DGF and rejection were similar between MP and static-storage kidneys on unadjusted analysis, on multivariable logistic regression adjusted for recipient and donor factors, recipients of MP kidneys were less likely to experience DGF and were less likely to experience rejection 1-year post-transplantation. This data suggests that MP should be a utilized in kidneys from selected donors.

Yarkın Kamil Yakupoğlu, MD

๑Copyright 2019 by the Association of Urological Surgery / Journal of Urological Surgery published by Galenos Publishing House. 